\title{
Retractile mesenteritis presenting as protein-losing gastroenteropathy
}

\author{
Bahe Rajendran MD, Donald R Duerksen MD
}

B Rajendran, DR Duerksen. Retractile mesenteritis presenting as protein-losing gastroenteropathy. Can J Gastroenterol 2006;20(12):787-789.

Retractile mesenteritis is a rare, idiopathic condition characterized by nonspecific inflammation of the mesenteric adipose tissue. The majority of patients present with abdominal pain and/or a palpable mass. In the present report, a 68-year-old man with peripheral edema and mild hypoalbuminemia is presented. Protein-losing gastroenteropathy was confirmed with an abnormal stool alpha ${ }_{1}$ antitrypsin clearance test and retractile mesenteritis was diagnosed at laparoscopy. This rare condition may respond to therapy with corticosteroids, azathioprine, cyclophosphamide, colchicine, progesterone, tamoxifen or thalidomide. Gastroenterologists should consider the diagnosis of protein-losing enteropathy in patients who present with unexplained peripheral edema or hypoalbuminemia. The test of choice to confirm this diagnosis is the stool alpha - $^{-}$ antitrypsin clearance test.

Key Words: Edema; Gastroenteropathy; Hypoalbuminemia; Retractile mesenteritis

$\mathrm{Q}$ etractile mesenteritis is a rare, idiopathic condition R.haracterized by nonspecific inflammation of the mesenteric adipose tissue. The majority of patients present with abdominal pain and/or a palpable mass (1). Fever, nausea, vomiting, diarrhea, anorexia, weight loss, fatigue and constipation are some of the other associated clinical symptoms.

In the present report, we describe a case of retractile mesenteritis in which the patient presented with chronic peripheral edema. This presentation has not been described before. In addition to emphasizing the importance of considering this diagnosis in cases of unexplained peripheral edema, we also reviewed

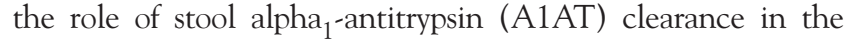
diagnosis of protein-losing gastroenteropathy (PLGE) and the value of diagnostic laparoscopy in further investigation of PLGE. Finally, the pathology, natural history and therapy of retractile mesenteritis is reviewed based on the reports of approximately 300 patient cases.

\section{CASE PRESENTATION}

A 68-year-old man was referred to the Saint Boniface General Hospital (Winnipeg, Manitoba) gastroenterology clinic for investigation of a possible gastrointestinal (GI) cause for an unexplained hypoalbuminemia and peripheral edema. His symptoms included chronic bilateral leg edema, nausea and mild change in bowel movements. He was having two to three loose, nonbloody stools per day, which was a change from having one formed stool per day. He had a two-year history of progressively

\author{
Mésentérite rétractile simulant une \\ entéropathie exsudative
}

La mésentérite rétractile est une maladie rare, idiopathique, qui se caractérise par une inflammation aspécifique du tissu adipeux du mésentère. La plupart des patients présentent des douleurs abdominales ou une masse palpable. Voici le cas d'un homme de 68 ans, qui présentait de l'œè̀me périphérique et une légère hypoalbuminurie. L'entéropathie exsudative a été confirmée par un test anormal de clairance de l'alpha-1-antitrypsine dans les selles, et la mésentérite rétractile a été confirmée par laparoscopie. Cette maladie rare peut se traiter par les corticostéroïdes, l'azathioprine, le cyclophosphamide, la colchicine, la progestérone, le tamoxifène ou la thalidomide. Les gastro-entérologues devraient envisager le diagnostic d'entéropathie exsudative chez les patients qui présentent de l'œdème périphérique d'origine inconnue ou de l'hypoalbuminurie. Le meilleur moyen de confirmer la présence de la maladie est le test de clairance de l'alpha-1-antitrypsine dans les selles. 


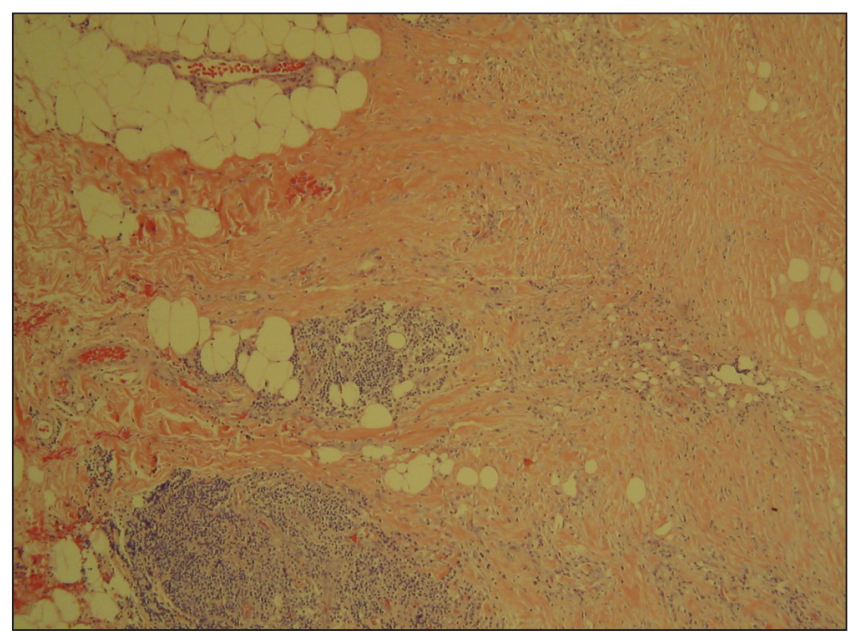

Figure 1) Mesenteric biopsy - low-power view showing fat necrosis, chronic inflammatory cells and fibrosis

Routine laboratory studies returned the following results: hemoglobin $150 \mathrm{~g} / \mathrm{L}$, white blood cell count $6.5 \times 10^{9} / \mathrm{L}$, platelet count $312 \times 10^{9} / \mathrm{L}$, urea $7.9 \mathrm{mmol} / \mathrm{L}$, creatinine $110 \mu \mathrm{mol} / \mathrm{L}$, alanine aminotransferase $23 \mathrm{U} / \mathrm{L}$, aspartate aminotransferase $18 \mathrm{U} / \mathrm{L}$, international normalized ratio 1.08 and thyroid stimulating hormone $2.6 \mathrm{mU} / \mathrm{L}$. Results of a screen for autoimmune disorders, including assessment of antinuclear antibody, rheumatoid factor and complement levels, were normal. Before his assessment at the Saint Boniface General Hospital gastroenterology clinic, the patient had undergone extensive cardiac investigations, including the multiple gated acquisition scan and echocardiography. His ejection fraction was $70 \%$ on the echocardiogram, with mild mitral valve and tricuspid valve regurgitations. A duplex ultrasound study ruled out the presence of deep vein thrombosis. His initial urinalysis showed $0.3 \mathrm{mg} / \mathrm{L}$ proteinuria with no abnormal cells. Further evaluation with a $24 \mathrm{~h}$ urine study (normal protein excretion of $130 \mathrm{mg} /$ day) and an abdominal ultrasound was unremarkable.

A $72 \mathrm{~h}$ fecal fat study was normal. An extensive upper and lower GI investigation, including gastroscopy, colonoscopy, small bowel follow-through and gastric emptying study, was unremarkable, except for some evidence of chronic gastritis and mild delay in gastric emptying. Duodenal, ileal and colonic biopsies were normal. A computed tomography scan of the abdomen showed no evidence of malignancy or inflammatory bowel disease, but multiple calcified mesenteric lymph nodes were thought to be related to a remote granulomatous disease.

Despite high protein intake, his serum total protein level was low $(58 \mathrm{~g} / \mathrm{L}$; normal $60 \mathrm{~g} / \mathrm{L}$ to $80 \mathrm{~g} / \mathrm{L})$ and serum albumin level was marginally low ( $28 \mathrm{~g} / \mathrm{L}$; normal $33 \mathrm{~g} / \mathrm{L}$ to $50 \mathrm{~g} / \mathrm{L})$. Because he had mild GI symptoms and no cause for his hypoalbuminemia was found, PLGE was considered to be a possibility. The $24 \mathrm{~h}$ fecal A1AT clearance was $165 \mathrm{~mL} / 24 \mathrm{~h}$ (normal less than $27 \mathrm{~mL} / 24 \mathrm{~h}$, Mayo Clinic Laboratory [USA]) which was consistent with a PLGE.

To investigate the possible cause of PLGE, the patient underwent a repeat gastroscopy with gastric and duodenal biopsies, which were normal. Because no obvious reason for the PLGE was found on the endoscopic and radiological investigations, a laparotomy was considered (2). Because of its less invasive nature, an exploratory laparoscopy was performed, which demonstrated

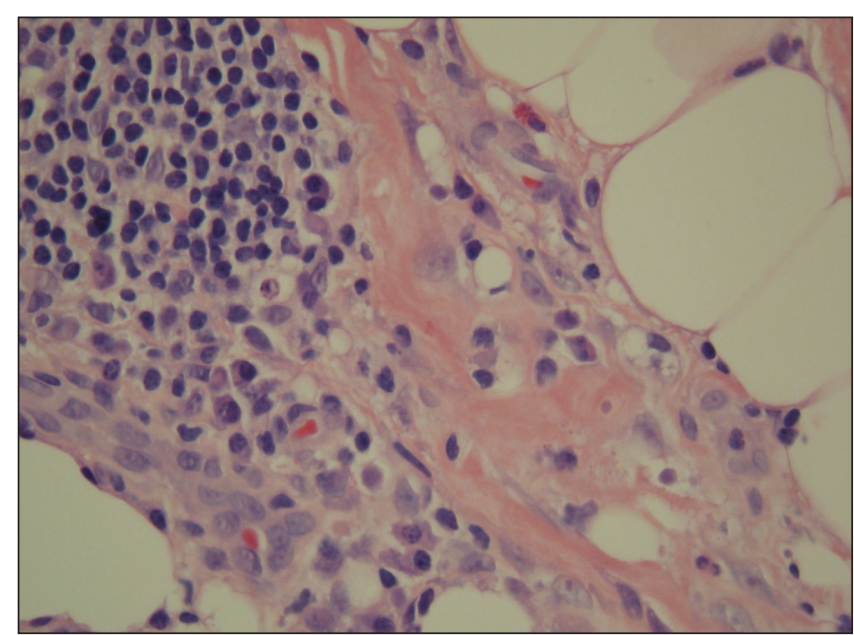

Figure 2) Mesenteric biopsy - high-power view showing fat necrosis, chronic inflammation and fibrosis

chronic inflammation of the mesentery, the presence of chylous ascites with dilated lymphatic channels, and multiple soft tissue masses in the abdominal wall and the mesentery. Mesenteric tissue biopsy showed fat necrosis with chronic inflammation and fibrosis; the histological findings were consistent with retractile mesenteritis (Figures 1 and 2). Peritoneal fluid analysis revealed an increased protein concentration of $22 \mathrm{~g} / \mathrm{L}$ and an albumin concentration of $14 \mathrm{~g} / \mathrm{L}$.

The patient was started on a course of prednisone $60 \mathrm{mg} /$ day for six weeks and exhibited some improvement in edema and nausea, but developed significant general weakness, shakiness and mood changes. His prednisone dose was tapered to $10 \mathrm{mg} /$ day. Colchicine $0.6 \mathrm{mg} /$ day was tried with no added benefit. Azathioprine was started at $100 \mathrm{mg} /$ day and gradually increased to $200 \mathrm{mg} /$ day, but despite this dose, adequate serum levels of 6-thioguanine nucleotide were not attained and the medication was discontinued. Prednisone was also discontinued. During the course of treatment, no improvements in the serum levels of albumin and protein were noticed. A subsequent course of cyclophosphamide and thalidomide was not effective in terms of symptomatic or biochemical improvement.

\section{DISCUSSION}

The 68-year-old man who presented with chronic leg edema and altered bowel habit was subsequently diagnosed with PLGE and retractile mesenteritis. On initial presentation, he had mild, nonspecific GI symptoms but progressively worsening leg edema. Before his extensive GI tract workup, he had undergone investigations to exclude cardiac, renal and liver disease. In addition, mucosal disease of the GI tract had been excluded. The diagnostic test which implicated the GI tract as a possible cause of his edema was the $72 \mathrm{~h}$ fecal A1AT clearance test.

Protein loss in the GI tract is a size-independent process that exhibits leakage of a wide spectrum of proteins, including immunoglobulins, ceruloplasmins, transferins, albumin and coagulation factors. Once serum proteins enter the GI tract, they are rapidly degraded into amino acids, limiting the value of stool detection tests. The gold standard for detecting enteric protein loss is measuring fecal loss of chromium 51-labelled albumin (3). This test is expensive and not readily available outside of research laboratories. The $72 \mathrm{~h}$ A1AT fecal protein clearance 
test is a simple test with a reasonable outcome. A1AT, which contributes $4 \%$ of the total protein, is resistant to proteolysis at a pH greater than 3, and is neither absorbed nor secreted. Measurement of fecal A1AT clearance has a sensitivity of $93.3 \%$ and a specificity of $90 \%$ compared with chromium 51 plasma protein clearance (4). Falsely elevated clearance may be seen in patients with chronic diarrhea and blood loss (3). Neither of these were evident in our patient. Random stool A1AT levels are insensitive in the diagnosis of PLGE (3). This test is not widely performed by laboratories in Canada but is available through the Calgary Laboratory Services (performed at the University of Alberta, Edmonton, Alberta).

There was only one other report of a patient with retractile mesenteritis presenting with PGLE (5). The patient had more overt malabsorption with increased fecal fat, and profoundly low albumin $(18 \mathrm{~g} / \mathrm{L})$ and total protein $(38 \mathrm{~g} / \mathrm{L})$ levels. A stool A1AT test was not performed.

Once PLGE is proven, the etiology needs to be identified. In our patient, mucosal diseases of the GI tract were ruled out by imaging studies and endoscopies with mucosal biopsies. Our case highlighted the value of diagnostic laparoscopy and biopsy in the diagnosis of the cause of PLGE. This was demonstrated in a previous case report (6).

Multiple terminologies have been used to describe inflammatory conditions of the mesentery. The most common terms are mesenteric lipodystropy, mesenteric panniculitis and sclerosing mesenteritis or retractile mesenteritis. Although these different terminologies have no clinical value, they are differentiated based on histological features. Emory et al (7) evaluated the mesenteric biopsies of 84 previously diagnosed patients for the three main histological features - fat necrosis, inflammation and fibrosis. Patients who had mesenteric lipodystrophy had focal and diffuse areas of fat necrosis with minimal or no inflammation and fibrosis. Those who were diagnosed with mesenteric panniculitis and retractile mesenteritis had predominantly chronic inflammation and fibrosis, respectively. The latter two groups also demonstrated mesenteric calcification in approximately $5 \%$ and $25 \%$ of the patients, respectively. However, there is significant overlap of the characteristic features among the three groups.

The evolution of this entity has been described in three stages. Stage 1 is mesenteric lipodystrophy, in which mesenteric

\section{REFERENCES}

1. Durst AL, Freund H, Rosenmann E, Birnbaum D. Mesenteric panniculitis: Review of the literature and presentation of cases. Surgery 1977;81:203-11.

2. Kim KE. Protein-losing gastroenteropathy. In: Feldman M, Friedman LS, Sleisenger MH, eds. Gastrointestinal and Liver Disease, 7th edn. Philadelphia: Saunders, 2002:446-52.

3. Strygler B, Nicar MJ, Santangelo WC, Porter JL, Fordtran JS. Alpha 1-antitrypsin excretion in stool in normal subjects and in patients with gastrointestinal disorders. Gastroenterology 1990;99:1380-7.

4. Florent C, L'Hirondel C, Desmazures C, Aymes C, Bernier JJ. Intestinal clearance of alpha 1-antitrypsin. A sensitive method for the detection of protein-losing enteropathy. Gastroenterology 1981;81:777-80.

5. Horing E, Hingerl T, Hens K, von Gaisberg U, Kieninger G. Protein-losing enteropathy: First manifestation of sclerosing mesenteritis. Eur J Gastroenterol Hepatol 1995;7:481-3.

6. Weiser J, Salky B, Slepian A, Dikman S. Laparoscopic diagnosis of retractile mesenteritis. Gastrointest Endosc 1992;38:615-7.

7. Emory TS, Monihan JM, Carr NJ, Sobin LH. Sclerosing mesenteritis, mesenteric panniculitis and mesenteric lipodystrophy: A single entity? Am J Surg Pathol 1997;21:392-8. fat is replaced by sheets of foamy macrophages. It is frequently an asymptomatic stage. Stage 2 is mesenteric panniculitis, characterized by inflammatory changes and lymphatic distension with early fibrosis. This may be the beginning of symptomatic presentation. Finally, stage 3 is retractile mesenteritis, in which collagen deposition and fibrosis thickens and shortens the mesentery. Eventually, these changes extend to the border of the small intestine, binding loops together which results in a palpable abdominal mass.

There are no randomized controlled trials of therapy for this condition. Medications that have been reported to improve symptoms and/or biochemistry include corticosteroids and azathioprine (8), cyclophosphamide (9), colchicine (10), progesterone (11) and tamoxifen (12). In a recent pilot study (13) with five symptomatic mesenteric panniculitis patients, thalidomide showed partial or complete response in four patients - one complete recovery in four weeks and three partial recoveries in 12 weeks. As with other immunosuppressive medications, thalidomide may be more effective in less fibrotic stages (mesenteric panniculitis) as opposed to retractile mesenteritis. This is considered to be a self-limiting entity and no early treatment is recommended (14), whereas others suggested aggressive, early treatment to prevent the progression of the disease (9). Antibiotics and irradiation treatments have been tried with no significant therapeutic value. Surgery is not indicated as a treatment option unless mechanical bowel obstruction or ischemia secondary to retraction and fibrous formation is identified.

\section{CONCLUSION}

The present case highlights the importance of considering the diagnosis of retractile mesenteritis in patients presenting with chronic peripheral edema that has no other identified cause on initial testing. The fecal A1AT clearance test is an inexpensive and reliable test of GI tract protein loss, and should be considered if unexplained GI protein loss is suspected. In cases in which the cause of PLGE is not identified after imaging and endoscopic evaluation, diagnostic laparoscopy should be considered. If steroids and other immunosuppressive and hormonal agents fail, a trial of thalidomide may be beneficial in treating this rare condition.

8. Tytgat GN, Roozendaal K, Winter W, Esseveld MR. Successful treatment of a patient with retractile mesenteritis with prednisone and azathioprine. Gastroenterology 1980;79:352-6.

9. Bush RW, Hammar SP Jr, Rudolph RH. Sclerosing mesenteritis. Response to cyclophosphamide. Arch Intern Med 1986;146:503-5.

10. Genereau T, Bellin MF, Wechsler B, et al. Demonstration of efficacy of combining corticosteroids and colchicine in two patients with idiopathic sclerosing mesenteritis. Dig Dis Sci 1996;41:684-8.

11. Oudemans-van Straaten HM, Jansen PG, Hoek FJ, et al. Intestinal permeability, circulating endotoxin, and postoperative systemic responses in cardiac surgery patients. J Cardiothorac Vasc Anesth 1996;10:187-94.

12. Venkataramani A, Behling CA, Lyche KD. Sclerosing mesenteritis: An unusual cause of abdominal pain in an HIV-positive patient. Am J Gastroenterol 1997;92:1059-60.

13. Ginsburg PM, Ehrenpreis ED. A pilot study of thalidomide for patients with symptomatic mesenteric panniculitis. Aliment Pharmacol Ther 2002;16:2115-22.

14. Koornstra JJ, van Olffen GH, van Noort G. Retractile mesenteritis: To treat or not to treat. Hepatogastroenterology 1997;44:408-10. 


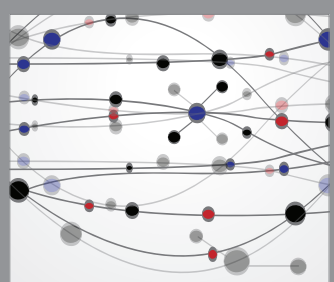

The Scientific World Journal
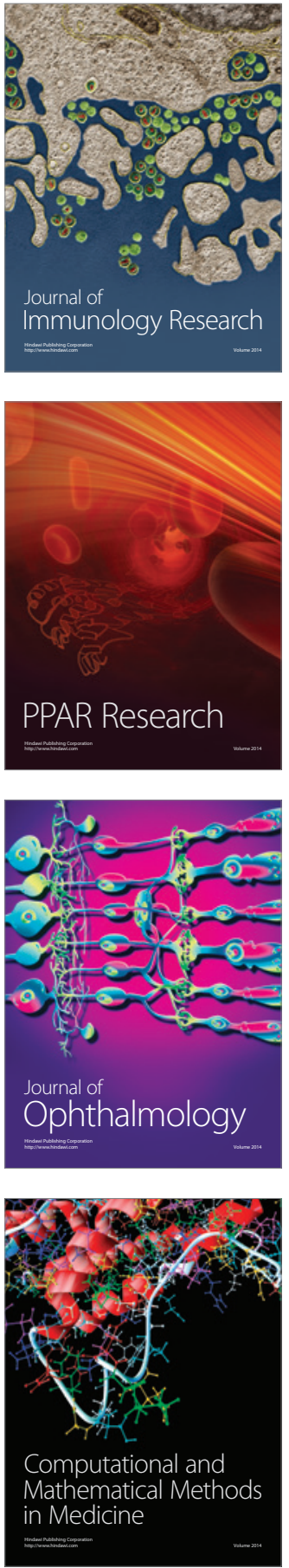

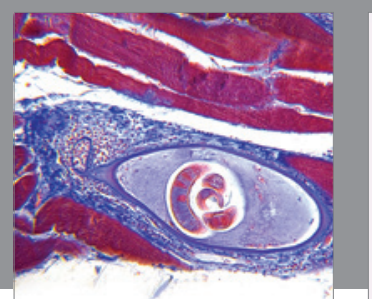

Gastroenterology Research and Practice

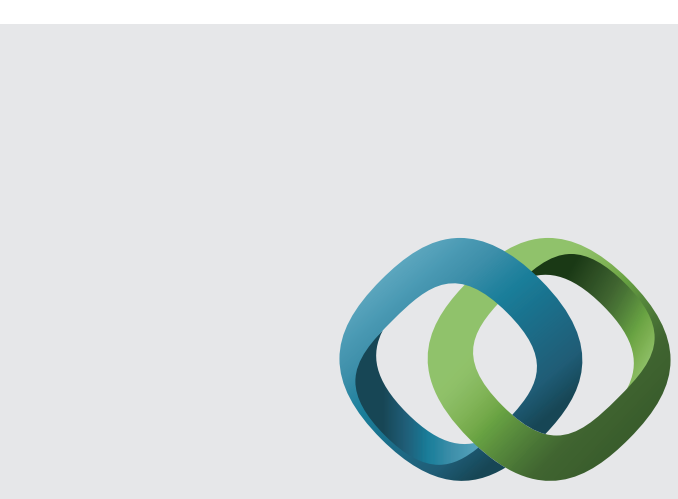

\section{Hindawi}

Submit your manuscripts at

http://www.hindawi.com
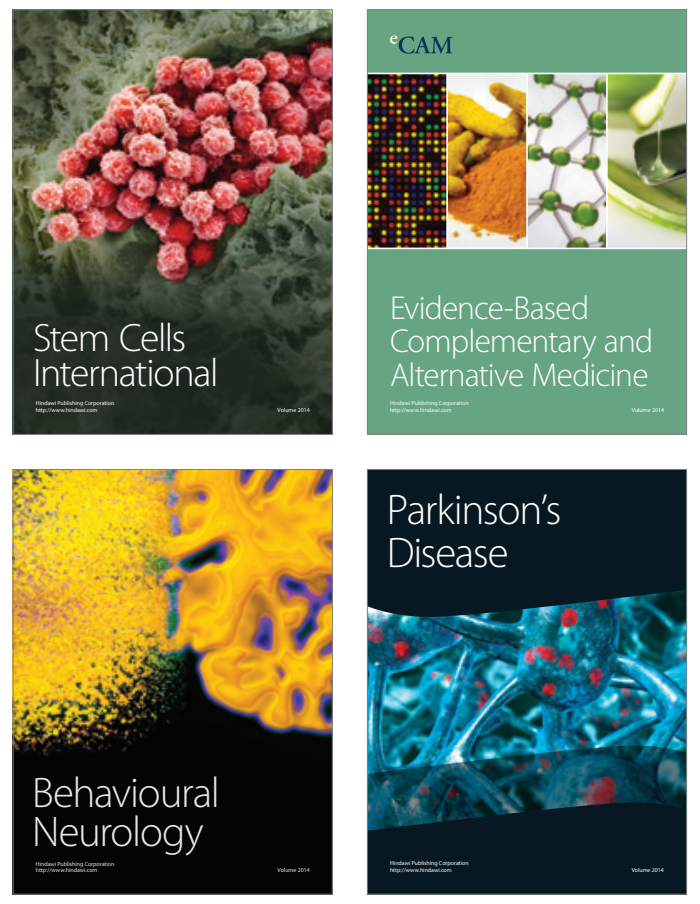
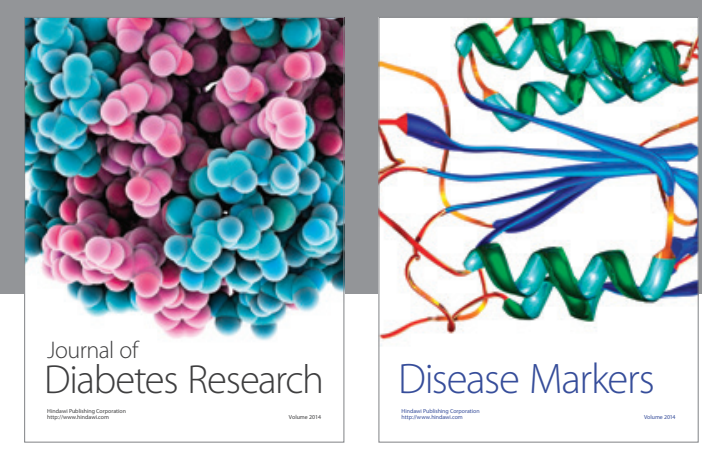

Disease Markers
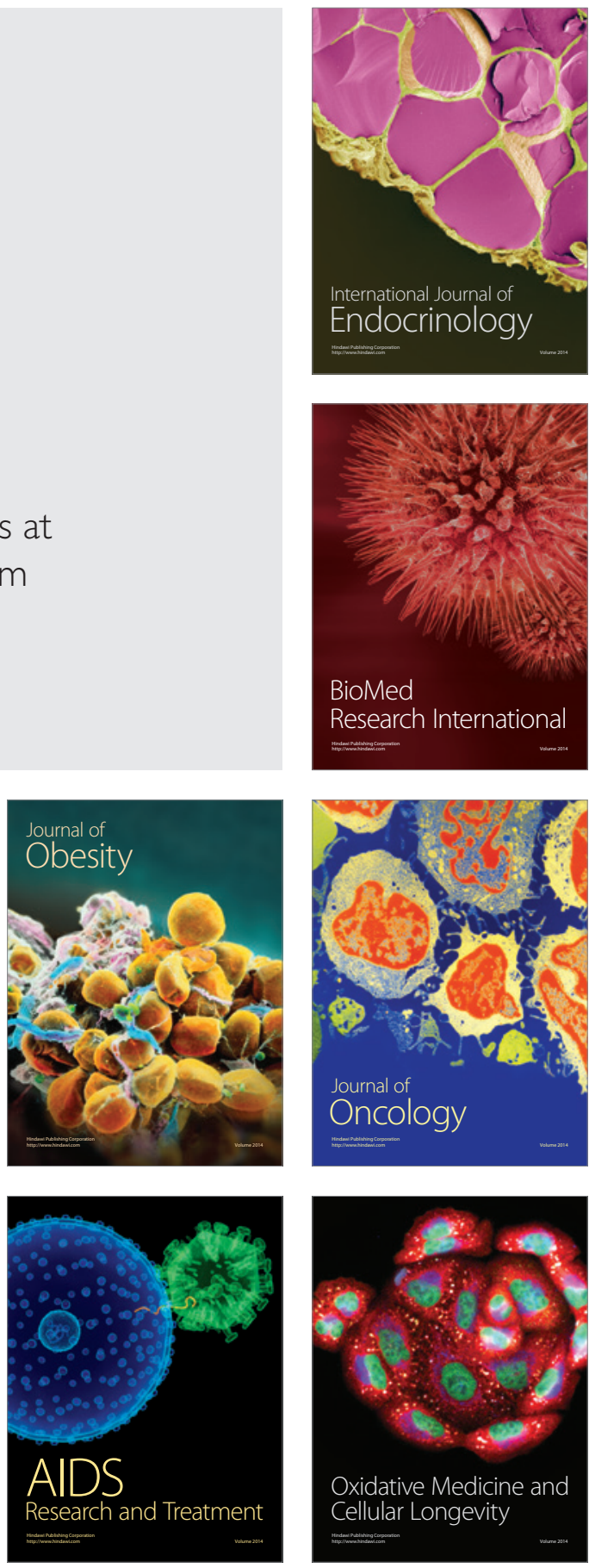